Hillebrecht, Lena

\title{
Entwicklung eines Modells zur Beurteilung der Qualität von berufsbegleitenden Studiengängen
}

Seifried, Jürgen [Hrsg.]; Seeber, Susan [Hrsg.]; Ziegler, Birgit [Hrsg.]: Jahrbuch der berufs- und wirtschaftspädagogischen Forschung 2016. Opladen; Berlin ; Toronto : Verlag Barbara Budrich 2016, S. 155-170. (Schriftenreihe der Sektion Berufs- und Wirtschaftspädagogik der Deutschen Gesellschaft für Erziehungswissenschaft (DGfE))

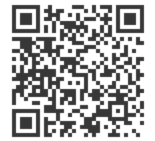

Quellenangabe/ Reference:

Hillebrecht, Lena: Entwicklung eines Modells zur Beurteilung der Qualität von berufsbegleitenden Studiengängen - In: Seifried, Jürgen [Hrsg.]; Seeber, Susan [Hrsg.]; Ziegler, Birgit [Hrsg.]: Jahrbuch der berufs- und wirtschaftspädagogischen Forschung 2016. Opladen; Berlin ; Toronto : Verlag Barbara Budrich 2016, S. 155-170 - URN: urn:nbn:de:0111-pedocs-127348 - DOI: 10.25656/01:12734

https://nbn-resolving.org/urn:nbn:de:0111-pedocs-127348

https://doi.org/10.25656/01:12734

in Kooperation mit / in cooperation with:

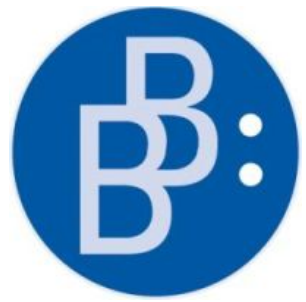

https://www.budrich.de

\section{Nutzungsbedingungen}

Dieses Dokument steht unter folgender Creative Commons-Lizenz: http://creativecommons.org/licenses/by-nc-nd/3.0/de/deed - Sie dürfen das Werk bzw. den Inhalt unter folgenden Bedingungen vervielfältigen, verbreiten und öffentlich zugänglich machen: Sie müssen den Namen des Autors/Rechteinhabers in der von ihm festgelegten Weise nennen. Dieses Werk bzw. dieser Inhalt darf nicht für kommerzielle Zwecke verwendet werden und es darf nicht bearbeitet, abgewandelt oder in anderer Weise verändert werden.

Mit der Verwendung dieses Dokuments erkennen Sie die Nutzungsbedingungen an.

\section{Terms of use}

This document is published under following Creative Commons-License: http://creativecommons.org/licenses/by-nc-nd/3.0/de/deed.en - You may copy, distribute and transmit, adapt or exhibit the work in the public as long as you attribute the work in the manner specified by the author or licensor. You are not allowed to make commercial use of the work or its contents. You are not allowed to alter, transform, or change this work in any other way.

By using this particular document, you accept the above-stated conditions of use.

\section{Kontakt / Contact:}

\section{peDOcs}

DIPF | Leibniz-Institut für Bildungsforschung und Bildungsinformation

Informationszentrum (IZ) Bildung

E-Mail: pedocs@dipf.de

Internet: www.pedocs.de

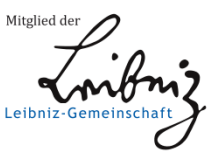




\section{Jahrbuch der berufs- und wirtschaftspädagogischen Forschung 2016}

Jürgen Seifried, Susan Seeber, Birgit Ziegler (Hrsg.)

DGE Deutsche Gesellschaft 
Schriftenreihe der Sektion

Berufs- und Wirtschaftspädagogik der Deutschen Gesellschaft

für Erziehungswissenschaft (DGfE) 
Jürgen Seifried

Susan Seeber

Birgit Ziegler (Hrsg.)

Jahrbuch der berufs- und

wirtschaftspädagogischen

Forschung 2016

Verlag Barbara Budrich

Opladen • Berlin • Toronto 2016 
Bibliografische Information der Deutschen Nationalbibliothek

Die Deutsche Nationalbibliothek verzeichnet diese Publikation in der Deutschen

Nationalbibliografie; detaillierte bibliografische Daten sind im Internet über

http://dnb.d-nb.de abrufbar.

(C) 2016 Dieses Werk ist im Verlag Barbara Budrich erschienen und steht unter folgender Creative Commons Lizenz: http://creativecommons.org/licenses/by-nc$\mathrm{nd} / 3.0 / \mathrm{de} /$

Verbreitung, Speicherung und Vervielfältigung erlaubt, kommerzielle Nutzung und Veränderung nur mit Genehmigung des Verlags Barbara Budrich

Dieses Buch steht im OpenAccess Bereich der Verlagsseite zum kostenlosen Download bereit (http://dx.doi.org/10.3224/84740588)

Eine kostenpflichtige Druckversion (Printing on Demand) kann über den Verlag bezogen werden. Die Seitenzahlen in der Druck- und Onlineversion sind identisch.

\section{ISBN 978-3-8474-0588-7 (Paperback) \\ eISBN 978-3-8474-0403-3 (eBook) \\ DOI $\quad 10.3224 / 84740588$}

Umschlaggestaltung: Bettina Lehfeldt, Kleinmachnow - www.lehfeldtgraphic.de Typographisches Lektorat: Angela Zerfaß, Leverkusen 


\section{Inhaltsverzeichnis}

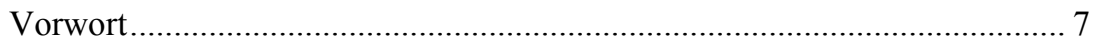

\section{Teil I: Organisation, Bedingungen und Strukturen der beruflichen Bildung}

Allison Fuller

Developing expertise: occupational versus job-based approaches in contemporary labour markets

Lorenz Lassnigg

„Duale“ oder „,dualistische“ Berufsbildung: Gemeinsamkeiten und

Unterschiede Österreich-Schweiz-Deutschland

Philipp Gonon und Lea Zehnder

Die Berufsbildung der Schweiz als permanenter

Kompromissbildungsprozess

Rolf Dobischat, Andy Schäfer, Christian Schmidt, Manfred Wahle und

Marcel Walter

Berufslaufbahnkonzepte: Instrumente zur Weiterentwicklung des

Berufsbildungssystems?...

Karl-Heinz Gerholz und Ursula Walkenhorst

Gestaltungsfragen zur Akademisierung der beruflichen Bildung am

Beispiel der Gesundheitsfachberufe. 73

\section{Teil II: Berufliche Lehr-Lern- und Unterrichtsforschung}

Juliana Schlicht

Handeln in Geschäftsprozessen als Forschungs- und

Lehr-Lern-Gegenstand

Alexandra Bach

Nutzung von digitalen Medien an berufsbildenden Schulen -

Notwendigkeit, Rahmenbedingungen, Akzeptanz und Wirkungen 107

Stefan Kessler

Branchen- und lernortspezifische Herausforderungen beim Einsatz von

Tablets in der überbetrieblichen Ausbildung der Schweizer Banken 125 


\section{Teil III: Hochschul- und Lehrerbildungsforschung}

Julia K. Gronewold

Struktur und Organisation berufsbegleitender MINT-Studiengänge

Lena Hillebrecht

Entwicklung eines Modells zur Beurteilung von Qualität in

berufsbegleitenden Studiengängen

Christoph Helm und Stefanie Antonia Mayer

Pedagogical Content Knowledge von Rechnungswesenlehrkräften -

Explorative Befunde einer Onlinebefragung aus Österreich

\section{Teil IV: Betriebliche Aus- und Weiterbildung}

Philipp Struck und Christian Dittmann

Weiterbildung im Handwerk. Rahmenbedingungen und

Handlungsfelder aus der Perspektive von Betrieben und Beschäftigten ..... 189

Andreas Rausch, Julia Warwas und Heike Jost

Die Beurteilung kaufmännischer Auszubildender in der betrieblichen

Praxis - Eine explorative Studie bei Industrie- und Bankkaufleuten

\section{Teil V: Historische Berufsbildungsforschung}

Frank Ragutt

Lesbarkeit der Branchen - Notizen zur Ordnungssemantik des Gewerbes in Realienbüchern des 19. Jahrhunderts.................................................... 223

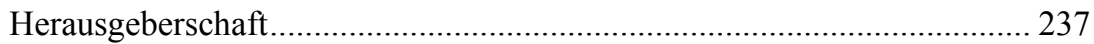

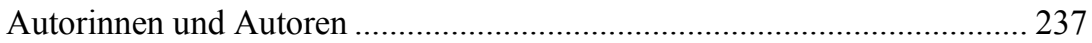




\title{
Entwicklung eines Modells zur Beurteilung der Qualität von berufsbegleitenden Studiengängen
}

\author{
Lena Hillebrecht
}

\section{Einleitung}

Die Bedeutung berufsbegleitender Bildungsangebote auf akademischem Niveau hat in den letzten Jahren zugenommen (vgl. Wolter \& Geffers 2013), jedoch nicht in dem Ausmaß, das mittels bildungspolitischer Initiativen wie der Hochschulöffnung für beruflich Qualifizierte (vgl. KMK 2009) beabsichtigt wurde. Es sollte die Studierendenquote unter Berufstätigen gesteigert werden, indem die Zahl der Studienberechtigten deutlich erhöht wurde (vgl. Wolter, Banscherus, Kamm \& Otto 2014). Zudem ist festzustellen, dass die Inanspruchnahme von Angeboten des Lebenslangen Lernens an Hochschulen in Deutschland im internationalen Vergleich eher gering ausfällt (vgl. Wolter et al. 2014). Ein möglicher Erklärungsansatz für die geringe Inanspruchnahme bestehender Studienangebote ist die Vermutung, dass die derzeit vorhandenen Studienmodelle aus Sicht der Studierenden nicht über eine ausreichende Passgenauigkeit und Qualität verfügen und daher für die Zielgruppe nicht ausreichend attraktiv sind (vgl. Wolter et al. 2014).

Gegenwärtig ist jedoch kein Instrument vorzufinden, das sich zur Beurteilung der Qualität dieser Studienmodelle einsetzen ließe, weshalb dessen Entwicklung und empirische Überprüfung erforderlich ist (vgl. Minks, Netz \& Völk 2011; Wolter et al. 2014). Ziel des in diesem Beitrag beschriebenen Vorhabens ist es daher, Studiengangsmerkmale zu identifizieren, die aus der Sicht der Studierenden für die Beurteilung von Qualität in berufsbegleitenden Studiengängen relevant sind. Diese Merkmale werden in einem Modell abgebildet, das mit einer quantitativen Studie überprüft wird. Für die Überprüfung wurde zunächst ein Fragebogen entwickelt, der eine Operationalisierung der Modellparameter darstellt. Anschließend wurde das so entwickelte Messinstrument im Rahmen einer quantitativen Erhebung pilotiert, um es anschließend in einer größeren Stichprobe einzusetzen. Im vorliegenden Beitrag liegt der Schwerpunkt auf der Darstellung der Ergebnisse der Modellpilotierung. 


\section{Forschungsstand}

Ausgangspunkt der Untersuchung von berufsbegleitenden Studiengängen bildet bei dem hier beschriebenen Vorhaben das „Rahmenmodell zur Wirkungsanalyse des Hochschulunterrichts“ von Helmke und Schrader (2006). Hierbei wird davon ausgegangen, dass die Effekte des Studiums, wie der Aufbau von Fachwissen oder Expertise, dadurch zustande kommen, dass die Lernenden auf Grundlage ihrer individuellen Studienbedingungen und Lernvoraussetzungen das Lehrangebot in einer individuellen Weise nutzen. Die Lehr-Lernsituation ist zudem eingebettet in den fachlichen, sozialen und ökologischen Kontext sowie die soziokulturellen Rahmenbedingungen (vgl. Helmke \& Schrader 2006).

Im vorliegenden Beitrag werden berufsbegleitende Studiengänge in den Blick genommen. Darunter werden Studiengänge verstanden, die von Berufstätigen absolviert werden und die speziell für diese Zielgruppe konzipiert wurden (vgl. Bargel \& Bargel 2014). Daher spielt auch die Zielgruppe und deren Belange eine wichtige Rolle bei der Beschäftigung mit diesen Studiengängen. Für die Beschreibung der Zielgruppe sind vor allem die Begriffe der nicht-traditionell Studierenden und der beruflich qualifizierten Studierenden heranzuziehen. Mit der ersten Bezeichnung sind Studierende gemeint, ,die nicht auf dem geraden Weg bzw. in der vorherrschenden zeitlichen Sequenz und Dauer zur Hochschule gekommen sind; nicht die regulären schulischen Voraussetzungen für den Zugang erfüllen; und solche, die nicht in der üblichen Form des Vollzeit- und Präsenzstudiums studieren (sondern als Teilzeit-, Abend- und Fernstudierende)“ (vgl. Teichler \& Wolter 2004).

Unter dem Begriff der beruflich Qualifizierten werden Personen gefasst, die eine berufliche Ausbildung erworben haben und zusätzlich eine Hochschulzugangsberechtigung besitzen oder Personen, die über den dritten Bildungsweg zum Studium gelangt sind (vgl. Autorengruppe Bildungsberichterstattung 2014). Es kann also davon ausgegangen werden, dass in berufsbegleitenden Studiengängen anteilig mehr Studierende bereits eine berufliche Vorbildung haben als in Vollzeit-Studiengängen. Folglich lässt sich festhalten, dass sich die Studierenden in berufsbegleitenden Studiengängen vor allem hinsichtlich ihrer Eingebundenheit durch berufliche Verpflichtungen und durch das häufige Vorhandensein beruflicher Vorqualifikationen auszeichnen. Es ist also davon auszugehen, dass sowohl Studierende mit einer schulischen, als auch Studierende mit einer beruflichen Hochschulzugangsberechtigung ein solches Studium aufnehmen (vgl. Wolter $\&$ Geffers 2013). Für die berufsbegleitend Studierenden ist folglich anzunehmen, dass es sich hierbei um eine Studierendengruppe mit stark ausgeprägter Heterogenität in Bezug auf die individuellen Lernvoraussetzungen 
handelt. Es kann angenommen werden, dass einige Studierende bereits über einschlägiges Vorwissen verfügen, das sie in einer beruflichen Vorbildung erworben haben. Defizite bzgl. der studienrelevanten Vorkenntnisse sind im Bereich Mathematik und beim wissenschaftlichen Arbeiten zu vermuten, was v. a. für Studierende gilt, die nicht über eine schulische Hochschulzugangsberechtigung verfügen (vgl. Wolter \& Geffers 2013).

Bezüglich der soziodemografischen Merkmale ist zu erwarten, dass die berufsbegleitend Studierenden im Mittel älter sind als diejenigen in VollzeitStudiengängen und dass sie häufiger bereits eine eigene Familie gegründet haben, was z.B. mit eingeschränkter örtlicher Mobilität und geringeren zeitlichen Ressourcen einhergehen kann, die zusätzlich durch die Berufstätigkeit eingeschränkt werden (vgl. Wolter \& Geffers 2013; Zinn 2012).

Bei berufsbegleitenden Studiengängen ist die Studieneingangsphase von besonderer Bedeutung. Hier sollten sowohl fachliche als auch strukturelle Beratungsangebote vorhanden sein (vgl. Minks et al. 2011), da vielfach von einer großen Unsicherheit bei der Entscheidung für ein Studium auszugehen ist (vgl. Arnold, Wetzel \& Dobmann 2014). Für die Entscheidung über die Aufnahme des Studiums ist es außerdem wichtig, dass die Durchlässigkeit zum Studium gewährleistet wird (vgl. Minks et al. 2011). Dies kann über die Schaffung flexibler Zugangswege zum Studium ermöglicht werden, was neben der Anerkennung von Abschlüssen auch die Gewährung von Anrechnungsoptionen für bereits erbrachte Leistungen (vgl. Wolter \& Geffers 2013) beinhalten kann. Außerdem kann die Durchlässigkeit durch Möglichkeiten zum Probestudium (vgl. Wolter 2000) oder Brückenkurse (vgl. Nickel \& Duong 2012) erhöht werden.

Es ist weiterhin davon auszugehen, dass die berufsbegleitend Studierenden nicht nur zu Beginn, sondern auch während des Studiums einen hohen Beratungsbedarf besitzen. Daher sollten für die Qualitätsbeurteilung auch die Beratungsleistungen der Hochschule während des Studiums berücksichtigt werden (vgl. Arnold et al. 2014).

Bei der Organisation des Studiums ist vermutlich eine flexible zeitliche Organisation bedeutsam, die Raum lässt für freie Zeiteinteilung durch die Lernenden (vgl. Schuetze \& Slowey 2000). Die Organisation in Teilzeit sollte so gestaltet sein, dass die Vereinbarkeit von Studium, Beruf und Privatleben gewährleistet wird (vgl. Schuetze \& Slowey 2000), was auch durch den Einbezug von geeigneten E-Learning-Sequenzen unterstützt werden kann (vgl. Schuetze \& Slowey 2000; Zawacki-Richter, von Prümmer \& Stöter 2015).

Der Zusammenhang zwischen Berufstätigkeit und Studienerfolg bzw. Studienzufriedenheit wurde insbesondere im angloamerikanischen Sprachraum untersucht. Riggert, Boyle, Petrosko, Ash und Parkins (2006) geben in ihrem Literature-Review einen Überblick über den Forschungsstand zur Thematik und schlussfolgern, dass die vorliegenden Studien zu uneinheitlichen 
Ergebnissen kommen: Die wöchentliche Arbeitszeit als Indikator für die berufliche Eingebundenheit hat in manchen Studien einen negativen, in anderen einen positiven Einfluss auf den Studienerfolg. Zudem stellen die Autoren heraus, dass kein theoretisches Modell zur Erklärung des Zusammenhangs zwischen Berufstätigkeit und Studienerfolg vorliegt. Anhaltspunkte hierfür lassen sich lediglich punktuell bei Modellen zur Erklärung des Studienabbruchs finden, in denen Art und Umfang der Berufstätigkeit als Einflussfaktor einbezogen werden (vgl. Riggert et al. 2006).

Weitere Erkenntnisse aus dem angloamerikanischen Raum liegen zu den Unterschieden zwischen nicht-traditionell Studierenden und den traditionell Studierenden vor. Donohue und Wong (1997) zeigten, dass bei nicht-traditionell Studierenden eine höhere Zielstrebigkeit im Studium zu beobachten ist. Es bleibt jedoch fraglich, ob Erkenntnisse aus dem angloamerikanischen Raum aufgrund der großen Unterschiede zwischen dem US-amerikanischen und dem deutschen Bildungssystem ohne Weiteres auf deutsche Studiengänge übertragen werden können (vgl. Zinn 2012).

Anknüpfungspunkte für die Untersuchung von berufsbegleitenden Studiengängen können bei Studien gewonnen werden, die Vollzeit-Studiengänge in den Blick nehmen, wie das SMILE-Projekt (vgl. Schiefele, Streblow, Ermgassen \& Moschner 2003) oder die Befragung der BA-Studierenden der FU Berlin (vgl. Thiel, Veit, Blüthmann, Lepa \& Ficzko 2008). Diese Studien liefern wichtige Erkenntnisse zu den Wirkungszusammenhängen zwischen Studienangebot, Studierendenmerkmalen und Studienerfolg im Allgemeinen. Es kann jedoch davon ausgegangen werden, dass bei berufsbegleitenden Studiengängen deren spezifische Besonderheiten zu berücksichtigen sind. Ähnlich gestaltete Studien wie die beiden hier exemplarisch angeführten wurden bisher noch nicht für berufsbegleitende Studiengänge durchgeführt.

Es lässt sich zusammenfassend festhalten, dass es einige Aspekte gibt, die für die Beurteilung von Qualität in berufsbegleitenden Studiengängen relevant erscheinen. Ein Modell, dass die empirische Untersuchung von Qualität ermöglichen könnte, liegt derzeit jedoch nicht vor (vgl. Wolter et al. 2014). 


\section{Fragestellung}

Das Ziel des hier beschriebenen Vorhabens ist es, ein Modell zu entwickeln und emprisch zu überprüfen, dass die Messung von Qualität in berufsbegleitenden Studiengängen ermöglichen soll. Wie bereits erläutert, liegt das Angebots-Nutzungs-Modell von Helmke und Schrader (2006) zugrunde. Das Modell bezieht sich in seiner Ausgestaltung durch die Autoren vordergründig auf einzelne Lehrveranstaltungen. In der hier beschriebenen Studie werden allerdings Studiengänge als Ganzes in den Blick genommen. Daher wird das Modell erweitert und auf der Angebotsseite wird nicht nur das Lehrangebot, sondern auch die strukturellen Studienbedingungen betrachtet. Dementsprechend wird bei der Betrachtung der Nutzung des Studienangebots sowohl die individuelle Nutzung des Lehrangebots, als auch die der organisatorischen Rahmenbedingungen berücksichtigt.

Zur Identifikation von relevanten Modellparametern bei berufsbegleitenden Studiengängen im Unterschied $\mathrm{zu}$ Vollzeit-Studiengängen wurde zunächst eine qualitative Studie durchgeführt, mittels derer Daten anhand von leitfadengestützten Experteninterviews mit drei verschiedenen Zielgruppen (Studierende: $n=7$, Hochschulmitarbeitende: $n=4$ und Arbeitgeber: $n=4$ ) erhoben wurden. Die Interviewtranskripte wurden im Zuge der zusammenfassenden Inhaltsanalyse nach Mayring (2010) ausgewertet, die Ergebnisse dieser Auswertung sind in dem nachfolgend beschriebenen Modell zusammengefasst.

Bei dem entwickelten Modell (Abb. 1) ist als abhängige Variable die Zufriedenheit der Studierenden mit den Gegebenheiten ihres Studiengangs gewählt. Dies soll die Beurteilung der Studienqualität durch die fokussierte Zielgruppe repräsentieren. 
Abb. 1: Modell zur Beurteilung von Qualität in berufsbegleitenden Studiengängen

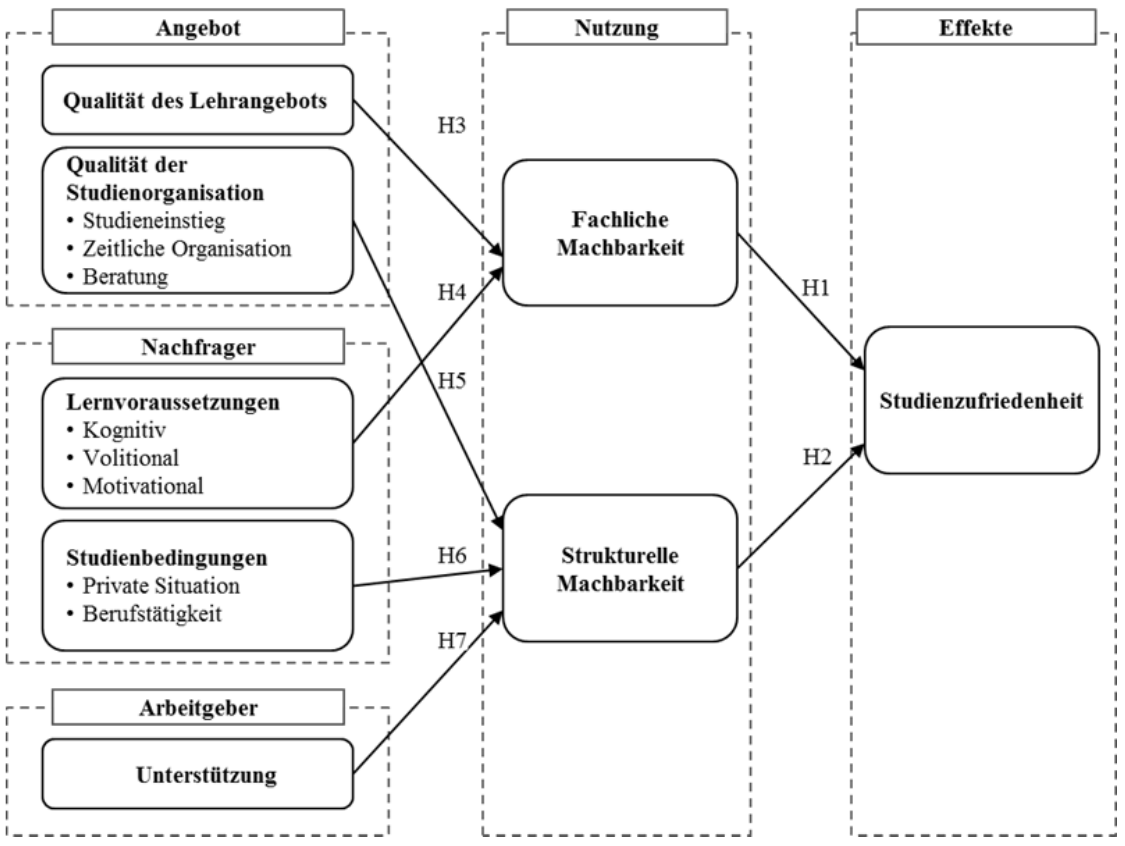

Quelle: eigene Abbildung

Dieses Qualitätsurteil wird im Modell auf zwei Aspekte zurückgeführt: die fachliche und die strukturelle Machbarkeit des Studiums. Gemeint ist, dass in diesen Machbarkeitsdimensionen die Studiengangsmerkmale auf die Studierendenmerkmale treffen und es $\mathrm{zu}$ einem bestimmten Grad an Passung zwischen den Merkmalen kommt, der zu einer fachlichen bzw. strukturellen Machbarkeit des Studiums aus Sicht des Studierenden führt. Demnach sind die Machbarkeitsdimensionen ein Ergebnis aus der Nutzung des Studienangebots auf Grundlage der Studierendencharakteristika. Aus diesem Modell ergeben sich die folgenden Forschungshypothesen.

H1: Ein hohes Maß an fachlicher Machbarkeit führt zu einer hohen Studienzufriedenheit.

H2: Ein hohes Maß an struktureller Machbarkeit führt zu einer hohen Studienzufriedenheit.

Durch diese Hypothesen kommt die Annahme zum Ausdruck, dass im berufsbegleitenden Studium sowohl die fachlichen Aspekte, die die eigentlichen Lehrveranstaltungen betreffen, als auch die organisationalen Rahmen- 
bedingungen einen Einfluss auf die Studienqualität haben. Das Zustandekommen der fachlichen Machbarkeit ist zum einen auf die Qualität des Lehrangebots zurückzuführen. Dieses Angebot wird von den Studierenden auf Grundlage ihrer kognitiven, volitionalen und motivationalen Lernvoraussetzungen individuell in Anspruch genommen. Es werden daher die folgenden Zusammenhänge angenommen:

H3: Je höher die Qualität des Lehrangebots, desto höher ist das Maß an fachlicher Machbarkeit.

H4: Je besser die individuellen Lernvoraussetzungen ausgeprägt sind, desto höher ist das Maß an fachlicher Machbarkeit.

Folglich lässt sich das Zustandekommen der fachlichen Machbarkeit so erläutern, dass die Nutzung des Lehrangebots durch die Studierenden unter Einfluss der individuellen Lernvoraussetzungen $\mathrm{zu}$ einem bestimmten Maß an fachlicher Passung führt.

Für die strukturelle Machbarkeit wird angenommen, dass diese insbesondere in berufsbegleitenden Studiengängen bedeutsam ist. Dies ist darauf zurückzuführen, dass die Studierenden durch ihre Berufstätigkeit und ihre private Situation stärker eingespannt sind und sich die Vereinbarkeit von Studium, Beruf und Privatem vermutlich schwieriger gestalten lässt, als es bei Vollzeitstudierenden üblicherweise der Fall ist. Daher wird davon ausgegangen, dass die strukturelle Machbarkeit einerseits von den Rahmenbedingungen des Studiengangs abhängt und andererseits die private Situation der Studierenden und die Unterstützung des Arbeitgebers in finanzieller, sozialer und zeitlicher Hinsicht eine Rolle spielen. Daher werden für die Erklärung der strukturellen Machbarkeit die folgenden Hypothesen zugrunde gelegt.

H5: Je besser die Qualität der organisationalen Rahmenbedingungen des Studiums, desto höher ist das Maß an struktureller Machbarkeit.

H6: Je stärker die Einbindung des Studierenden durch private und berufliche Verpflichtungen, desto geringer ist das Maß an struktureller Machbarkeit.

H7: Je höher das Maß an Unterstützung seitens des Arbeitgebers, desto höher ist das Maß an struktureller Machbarkeit.

Durch die Kombination von Aspekten der Studienorganisation, der individuellen Studienbedingungen sowie Aspekten der Berufstätigkeit bringt die strukturelle Machbarkeit als Unterfacette zum Ausdruck, inwiefern die Vereinbarkeit von Studium, Beruf und Privatem gelingt.

Es werden hier berufsbegleitende Studiengänge in den Blick genommen, die zu einem akademischen Abschluss führen und im wirtschaftswissen- 
schaftlichen Bereich angesiedelt sind. Die Betrachtungsebene ist der Studiengang als Ganzes. Es werden daher nicht einzelne Lehrveranstaltungen oder Phasen des Studiums betrachtet.

\section{Methodische Vorgehensweise}

Die methodische Vorgehensweise ist in zwei empirische Teilstudien gegliedert. Wie bereits in Abschnitt 3 angesprochen wurde, ist die qualitative Vorstudie, die erste Teilstudie, bereits abgeschlossen. Basierend auf den Ergebnissen der Vorstudie wurde die quantitativ angelegte Hauptstudie konzipiert.

Die in diesem Beitrag fokussierte Pilotierung der Hauptstudie soll dazu dienen, das zuvor ausgearbeitete Modell empirisch zu überprüfen. Dies geschieht im Rahmen einer quantitativen Fragebogenstudie bei berufsbegleitend Studierenden. Die Umfrage erfolgt als Online-Erhebung, mit einer ungefähren Testzeit von 30 Minuten. Im Fragebogen sind Skalen zu der Wahrnehmung der Studierenden der Lehrqualität und der Qualität organisatorischen Rahmenbedingungen enthalten. Darüber hinaus werden die individuellen Lernvoraussetzungen und die Intensität der ArbeitgeberUnterstützung erfasst. Soweit vorhanden, wurden bereits empirisch erprobte Skalen verwendet (Tab. 2). Zusätzlich zu den unten aufgeführten Skalen wurden Informationen zum besuchten Studiengang, $\mathrm{zu}$ den Vorqualifikationen, zur privaten Situation sowie zum sozioökonomischen Status und zur Berufstätigkeit erhoben.

Bei der Konstruktion der Skalen, die das Urteil der Studierenden über den besuchten Studiengang erfassen sollen, wurde darauf geachtet, dass subjektiven Erwartungen der Befragten die Antwort nicht beeinflussen. Die Pilotierung erfolgte im Oktober und November $2015(n=111)$. Die verwendeten Skalen werden gemäß den Ergebnissen der Analysen der Pilotierungsstichprobe für die Haupterhebung (erwartetes $n=400$ ) modifiziert. Mit der quantitativen Studie soll gezeigt werden, inwiefern sich das Forschungsmodell mithilfe des Fragebogeninstruments überprüfen lässt. Bei der Pilotierungsstudie ging es vordergründig darum, die Skalengüte zu überprüfen und zu untersuchen, ob die Modellannahmen grundsätzlich zu bestätigen sind. 
Tab. 1: Übersicht über die eingesetzten Skalen

\begin{tabular}{ll}
\hline Skala (mit Quelle) & Beispielitem \\
\hline $\begin{array}{l}\text { Qualität des Studieneinstiegs } \\
\text { Qualität der zeitlichen }\end{array}$ & $\begin{array}{l}\text { Es gab im Vorfeld Informationsveranstaltungen } \\
\text { über das Studium. }\end{array}$ \\
$\begin{array}{l}\text { Organisation } \\
\text { (Eigenentwicklung) }\end{array}$ & $\begin{array}{l}\text { Die Lehrveranstaltungen sind mit den } \\
\text { Selbststudienphasen zeitlich abgestimmt }\end{array}$ \\
$\begin{array}{l}\text { Beratungsqualität } \\
\text { (Eigenentwicklung) }\end{array}$ & $\begin{array}{l}\text { An meiner Hochschule stehen kompetente } \\
\text { Ansprechpartner für die Beratung zur }\end{array}$ \\
$\begin{array}{l}\text { Studienzufriedenheit } \\
\text { (Eigenentwicklung) }\end{array}$ & $\begin{array}{l}\text { Verfügung. } \\
\text { Wenn ich noch einmal vor der Wahl stehen }\end{array}$ \\
$\begin{array}{l}\text { Lehrqualität } \\
\text { (Adaption Schiefele, Moschner }\end{array}$ & $\begin{array}{l}\text { In den meisten Lehrveranstaltungen gelingt es } \\
\text { den Lehrenden, den Lernstoff gut zu erklären. }\end{array}$ \\
$\begin{array}{l}\text { \& Husstegge, 2002) } \\
\text { Studienmotivation } \\
\text { (Wild, Krapp, Schiefele, }\end{array}$ & $\begin{array}{l}\text { Ich lerne im Studium, weil ich mein Studium } \\
\text { erfolgreich abschließen möchte. }\end{array}$ \\
$\begin{array}{l}\text { Lewalter \& Schreyer, 1995) } \\
\text { Akademisches Selbstkonzept } \\
\text { (Marsh \& O’Neill, 1984, in der } \\
\text { dt. Fassung nach Hußtegge, }\end{array}$ & $\begin{array}{l}\text { In den meisten Lehrveranstaltungen kann ich } \\
\text { mich auf meine Begabung verlassen }\end{array}$ \\
$\begin{array}{l}\text { 2001) } \\
\text { Intensität der Arbeitgeber- }\end{array}$ & $\begin{array}{l}\text { Die Vereinbarkeit von Studium und Beruf wird } \\
\text { pnterstützung }\end{array}$ \\
$\begin{array}{l}\text { pigenentwitiv durch die Unterstützung meines } \\
\text { Arbeitgebers beeinflusst. }\end{array}$ \\
\hline
\end{tabular}

Quelle: Eigene Abbildung

\section{Ergebnisse der Pilotierungsstudie}

Im Folgenden wird auf die Ergebnisse der Pilotierung der Modellüberprüfungsphase eingegangen. Zunächst wird die Zusammensetzung der Stichprobe mit deskriptiven Statistiken beschrieben, die mithilfe von SPSS statistics 23 berechnet wurden. Das Forschungsmodell wurde mithilfe von Strukturgleichungsmodellierung überprüft, wofür das PLS-Verfahren eingesetzt wurde, da es die Möglichkeit bietet, komplexe Zusammenhänge auch bei kleinen Stichprobengrößen zu untersuchen (vgl. Ringle, Sarstedt \& Straub 2012). Die Ergebnisdarstellung nimmt als erstes auf das Messmodell und dann auf das Strukturmodell Bezug. Die zugrundeliegenden Analysen erfolgten mit der Software SmartPLS 2.0. 


\subsection{Beschreibung der Stichprobe}

Die Pilotierung der Messinstrumente erfolgte mit einer Online-Erhebung bei berufsbegleitend Studierenden ( $\mathrm{n}=111$ ). Die Stichprobe setzt sich zu 59\% aus männlichen und zu 38\% aus weiblichen Studierenden zusammen. Studierende, die an Universitäten studieren, machen etwa ein Viertel der Stichprobe aus $(26 \%$ ), die restlichen $74 \%$ besuchten einen Studiengang an einer (Fach-) Hochschule. Insgesamt sind Beobachtungen von zehn Hochschulen und Universitäten im Datensatz enthalten. Von den $68 \%$ der Studierenden, die einen Bachelorabschluss anstreben, befinden sich die meisten im dritten bis fünften Fachsemester. Bei den 24\% Masterstudierenden ist die Fachsemesterzahl sehr heterogen. Keinen Bachelor- oder Masterabschluss, sondern einen MBA-Abschluss, strebten 8\% der Probanden an. Alle untersuchten Studiengänge sind in den Wirtschaftswissenschaften angesiedelt.

\subsection{Messmodell}

Um zu zeigen, inwiefern die Daten das Modell abbilden können, soll nun die Validität des Messmodells untersucht werden. Dafür werden konvergente und die diskriminante Validität in den Blick genommen (siehe Tabelle 2).

Alle standardisierten Faktorladungen liegen nahe bei oder über dem Grenzwert von .70, was für eine hohe Indikatorreliabilität spricht (vgl. Hulland 1999). Die verwendeten Skalen weisen ein Cronbach's Alpha und eine Composite Reliability von größer .70 auf, so dass die Reliabilität als gut bewertet werden kann (vgl. Bagozzi \& Yi 1988). Anhand der Tatsache, dass bei allen Skalen die durchschnittlich erfasste Varianz über .5 liegt (vgl. Tab. 3), lässt sich eine gute Konvergenzvalidität feststellen (vgl. Bagozzi \& Yi 1988).

Die Ladung der Items zu ihrem zugehörigen Faktor ist jeweils höher als die $\mathrm{zu}$ allen anderen Faktoren, so dass auf ein ausreichendes Maß an diskriminante Validität zu schließen ist (vgl. Chin 1998). Das Messmodell erfüllt somit die Qualitätskriterien und kann für weitere Analysen verwendet werden.

Tab. 2: Kennwerte zu den eigesetzten Skalen

\begin{tabular}{lccccc}
\hline & AVE & CR & CA & MW & SD \\
\hline Lehrqualität & 0.694 & 0.872 & 0.779 & 3.033 & 0.430 \\
Einstiegs-Qualität & 0.656 & 0.884 & 0.825 & 3.049 & 0.618 \\
Zeitmodell-Qualität & 0.656 & 0.850 & 0.750 & 3.131 & 0.583 \\
Beratungs-Qualität & 0.621 & 0.867 & 0.796 & 3.069 & 0.589 \\
\hline
\end{tabular}


Tab. 2: Kennwerte zu den eigesetzten Skalen (Foirtsetzung)

\begin{tabular}{lccccc}
\hline & AVE & CR & CA & MW & SD \\
\hline Extrinsische Motivation & 0.597 & 0.880 & 0.847 & 2.753 & 0.632 \\
Intrinsische Motivation & 0.756 & 0.903 & 0.840 & 2.935 & 0.669 \\
Akadem. Selbstkonzept & 0.614 & 0.888 & 0.843 & 2.784 & 0.530 \\
Intensität AG-Unterstützung & 0.740 & 0.895 & 0.827 & 2.517 & 0.868 \\
Fachliche Machbarkeit & 0.647 & 0.846 & 0.726 & 3.037 & 0.607 \\
Strukturelle Machbarkeit & 0.627 & 0.834 & 0.706 & 3.113 & 0.565 \\
Zufriedenheit & 0.706 & 0.906 & 0.862 & 3.241 & 0.654 \\
\hline
\end{tabular}

Bei allen Konstrukten wurden verbale Ratingskalen mit vier Kategorien verwendet (,,trifft gar nicht $z u$ “ bis ,,trifft voll zu“). AVE=average explained variance, durchschnittlich erklärte Varianz, $C R=$ Composite Reliability, $C A=$ Cronbach's Alpha, $M W=$ Mittelwert, $S D=$ Standardabweichung.

Quelle: eigene Abbildung

\subsection{Strukturmodell}

Um die Signifikanzniveaus des Strukturmodells ermitteln zu können, wurde die Bootstrapping Re-Sampling Methode verwendet, mit der 5000 Stichproben erzeugt wurden (vgl. Hair, Ringle \& Sarstedt 2011). Den Analysen wird ein Signifikanzniveau von 10\% zugrunde gelegt, da im Rahmen der Pilotierung lediglich eine kleine Stichprobe erhoben wurde. Abb. 2 zeigt die mithilfe des PLS-Verfahrens geschätzten Pfadkoeffizienten sowie die mit dem Bootstrapping ermittelten Signifikanzniveaus.

Bezogen auf die formulierten Hypothesen können die folgenden Ergebnisse festgehalten werden: Sowohl $\mathrm{H} 1$ als auch $\mathrm{H} 2$ können bestätigt werden, denn sowohl die fachliche als auch die strukturelle Machbarkeit haben einen signifikant positiven Einfluss auf die Gesamtzufriedenheit mit dem Studium. Zudem zeigt es sich, dass die Zufriedenheit zu einem großen Anteil $\left(\mathrm{R}^{2}=.485\right)$ mithilfe der beiden Machbarkeitsdimensionen erklärt werden kann.

H3 beinhaltete die Annahme, dass die fachliche Machbarkeit auf die Qualität des Lehrangebots zurückzuführen sei. Dieser positiv angenommene Zusammenhang kann bestätigt werden, da die Lehrqualität einen signifikanten positiven Einfluss auf die fachliche Machbarkeit hat.

Der in $\mathrm{H} 4$ formulierte Zusammenhang der fachlichen Machbarkeit mit den individuellen Lernvorrausetzungen lässt sich mit dem empirischen Modell nur zum Teil bestätigen. Das akademische Selbstkonzept hat einen positiven, signifikanten Einfluss, die intrinsische sowie die extrinsische 
Motivation haben jedoch keinen signifikanten Einfluss. Auch für die letzte, schulisch erworbene Mathematiknote als Indikator für die kognitiven Lernvoraussetzungen zeigt sich kein signifikanter Einfluss auf die fachliche Machbarkeit. Folglich kann H4 nur für das akademische Selbstkonzept bestätigt werden, da lediglich hier ein signifikanter Zusammenhang vorliegt.

Abb. 2: Empirisches Modell

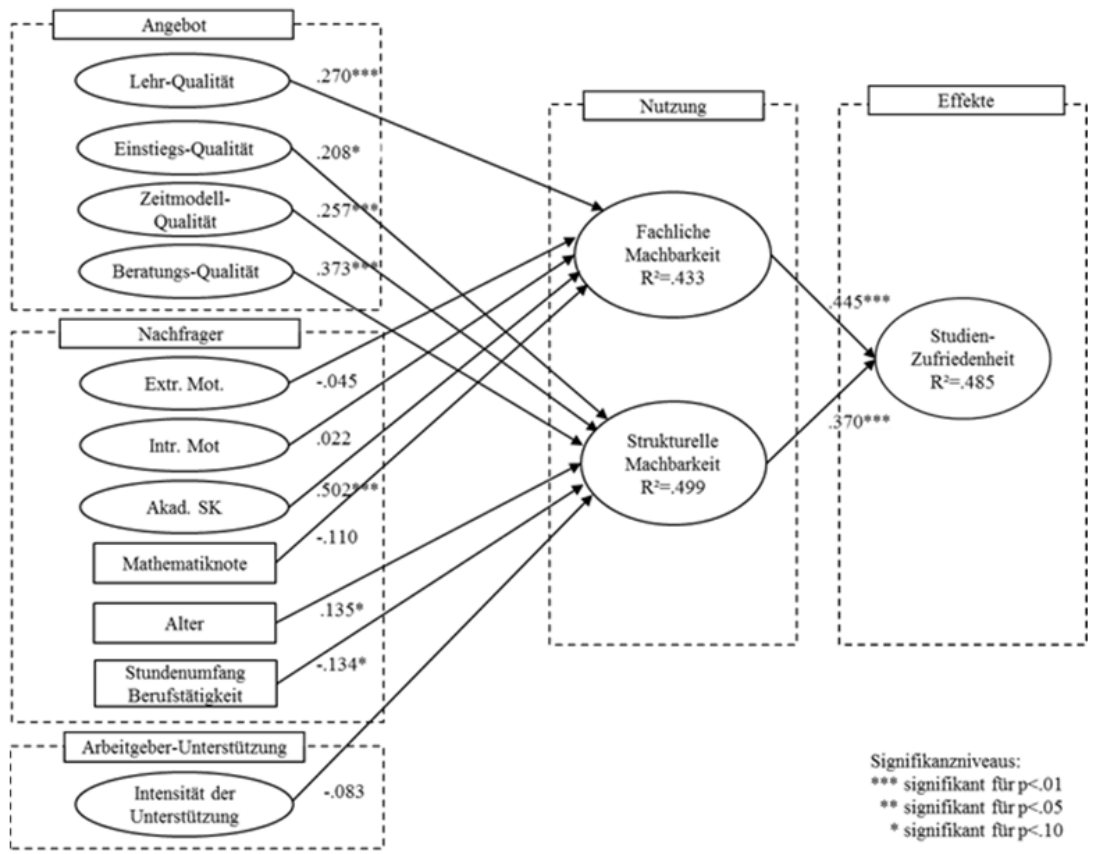

Quelle: eigene Abbildung

Der in H5 angenommene positive Zusammenhang zwischen der wahrgenommenen Qualität der Studienbedingungen und der strukturellen Machbarkeit kann für die Qualität des Studieneinstiegs, des Zeitmodells und der Beratung bestätigt werden. Es kann also davon ausgegangen werden, dass sich die strukturelle Machbarkeit umso besser gestaltet, je besser die Qualität der Studienorganisation wahrgenommen wird.

Zu H6 lässt sich sagen, dass das Alter der Studierenden einen signifikant positiven Einfluss und der wöchentliche Stundenumfang der Berufstätigkeit einen signifikant negativen Einfluss auf die strukturelle Machbarkeit hat. Für den Stundenumfang lässt sich H6 bestätigen, da dieser als Indikator für die Intensität der beruflichen Eingebundenheit gesehen wird. Je stärker die 
Studierenden beruflich eingespannt sind, desto schwieriger gestaltet sich die strukturelle Machbarkeit des Studiums. Für das Lebensalter wurde angenommen, dass ein höheres Lebensalter mit einer stärkeren familiären Eingebundenheit und diese mit einer schlechteren strukturellen Machbarkeit einhergeht. Die Ergebnisse zeigen jedoch den umgekehrten Zusammenhang, so dass H6 nur für die berufliche Eingebundenheit bestätigt werden kann. H7 kann nicht bestätigt werden, da kein signifikanter Einfluss der Intensität der Arbeitgeber-Unterstützung auf die strukturelle Machbarkeit vorliegt.

\section{Diskussion und Ausblick}

Obwohl die Bedeutung berufsbegleitender Studiengänge in den vergangenen Jahren gestiegen ist, bleiben die Studierendenzahlen hinter den Erwartungen zurück. Ein Ansatz, der Erklärungen für die verhältnismäßig geringen Studierendenzahlen liefern kann, ist es, zu untersuchen, wie die Qualität der derzeitigen Studienangebote im berufsbegleitenden Bereich durch die Studierenden beurteilt wird.

Der vorliegende Beitrag liefert daher einen Modellentwurf zur Überprüfung von Qualität in berufsbegleitenden Studiengängen. Dabei finden die durch die Studierenden beurteilte Qualität der Studienorganisation und der Lehrveranstaltungen Berücksichtigung. Deren Einfluss auf die Qualität wird im Zusammenwirken mit individuellen Studierendenmerkmalen und Aspekten der Berufstätigkeit untersucht.

Die Ergebnisse der Pilotierung zeigen, dass die eingesetzten Skalen die Anforderungen hinsichtlich der Gütekriterien erfüllen und für eine weitere Verwendung geeignet erscheinen. Die Ergebnisse bezüglich des Strukturmodells legen nahe, dass die getroffenen Modellannahmen grundsätzlich bestätigt werden können. Die Studienzufriedenheit lässt sich mit den gewählten Teildimensionen fachliche und strukturelle Machbarkeit erklären und die Teildimensionen selbst können mit den Merkmalen des Studiums, der Studierenden und der Berufstätigkeit zu einem hohen Anteil erklärt werden.

Es ist jedoch erkennbar, dass der Einfluss der Studiengangsmerkmale auf die Machbarkeitsdimensionen stärker ist als jener der Studierendenmerkmale, da hier häufiger signifikante Zusammenhänge vorliegen. Eine mögliche Erklärung hierfür ist, dass in den Machbarkeitsdimensionen bereits die Passung zwischen Angebot und Nachfrager enthalten ist und daher die Studiengangskonzeption insoweit auf die Charakteristika der Zielgruppe ausgerichtet ist, dass eine Passung vor allem von ihrer Ausgestaltung bestimmt wird. Weitere Analysen könnten zeigen, welche Studienmodelle zu einer besonders guten Passung führen, so dass Spielräume der Studiengangsentwicklung in Hinblick auf die Machbarkeit des Studiums identifiziert 
werden können. Auf Basis der geringen Beobachtungszahl in der Pilotierungsstichprobe ist dies noch nicht möglich. Es konnten bisher auch nicht alle theoretisch identifizierten Zusammenhänge bestätigt werden. Eine wesentliche Erkenntnis war die Annahme, dass bei berufsbegleitenden Studiengängen neben fachlichen Aspekten auch strukturelle Aspekte sehr bedeutsam für die Qualitätsbeurteilung sind. Dies lässt sich anhand der Ergebnisse bestätigen.

Im dargestellten Modell werden einige mögliche Zusammenhänge zwischen Modellparametern nicht berücksichtigt. Dies gilt z.B. für den Zusammenhang zwischen den kognitiven Lernvoraussetzungen und der strukturellen Machbarkeit des Studiums. Bei den hier beschriebenen Untersuchungen wurde eine Konzentration auf die Haupteffekte versucht, auch wenn Zusammenhänge zwischen fachlichen und strukturellen Aspekte auch denkbar sind.

Bei der Untersuchung der Qualität des Studieneinstiegs wird lediglich die Perspektive der Studierenden berücksichtigt, die tatsächlich ein Studium aufgenommen haben. Es haben jedoch vermutlich weitere Personen Angebote zum Studieneinstieg in Anspruch genommen, sich dann aber doch gegen ein Studium entscheiden. Eine umfassende Untersuchung des Studieneinstiegs müsste das Urteil aller Studieninteressierten berücksichtigen. Dies ist jedoch aus forschungsmethodischer Sicht problematisch, insbesondere aufgrund der schwierigen Kontaktaufnahme, weshalb von einem Einbezug dieser Personen abgesehen wurde. Lohnenswert wäre es auch, die Studierenden über mehrere Semester hinweg mit einer längsschnittlichen Studie zu begleiten. So könnten die Wirkungszusammenhänge zwischen den Studiengangs- und Studierendenmerkmalen detaillierter untersucht werden.

In dem hier beschriebenen Vorhaben werden lediglich wirtschaftswissenschaftliche Studiengänge in den Blick genommen, so dass offen bleibt, ob die Ergebnisse auch für andere Fachrichtungen gelten. Es zeigte sich aber, dass dies bei den angebotenen berufsbegleitenden Studiengängen die am häufigsten vertretene Fachrichtung ist (vgl. Minks et al. 2011). Folglich können die hier gewonnenen Erkenntnisse zwar nicht für alle in Deutschland angebotenen berufsbegleitenden Studiengänge gelten, wohl aber für eine große Gruppe.

Es wird sich zeigen, inwiefern die gefundenen Ergebnisse auch im Zuge der Haupterhebung bei einer größeren Stichprobe (erwartetes $n=400$ ) Gültigkeit aufweisen und inwiefern noch weitere Erkenntnisse zur Erklärung der Studienqualität gewonnen werden können. Anhand dieser Daten sollen die Modellzusammenhänge mit weiterführenden Analysen untersucht werden. Ein Ansatzpunkt hierfür ist der Einbezug weiterer Studiengangsmerkmale, um z.B. Unterschiede zwischen Bachelor- und Masterstudiengängen $\mathrm{zu}$ untersuchen. Es könnten auch Mehrebenenanalysen durchgeführt werden, durch die die gruppierte Struktur der Daten 
berücksichtigt werden kann, sofern die Datenstruktur dies zulassen wird. Darüber hinaus sind weiterführende Analysen zur Wichtigkeit der Faktoren untereinander sowie eine Potenzialanalyse geplant.

\section{Literatur}

Arnold, M., Wetzel, K. \& Dobmann, B. (2014). Erwartungen an die Qualität berufsbegleitender Studiengänge aus Hochschul-und Unternehmensperspektive - eine vergleichende Untersuchung. Beiträge zur Hochschulforschung, 36(4), 64-91.

Autorengruppe Bildungsberichterstattung (Hrsg.) (2014). Bildung in Deutschland 2014. Ein indikatorengestützter Bericht mit einer Analyse zur Bildung von Menschen mit Behinderungen. Bielefeld: Bertelsmann.

Bagozzi, R. P., \& Yi, Y. (1988). On the evaluation of structural equation models. Journal of the Academy of Marketing Science, 16(1), 74-94.

Bargel, T. \& Bargel, H. (2014). Studieren in Teilzeit und Teilzeitstudium. Bielefeld: Universitätsverlag Webler.

Chin, W. W. (1998). The partial least squares approach to structural equation modeling. In G. A. Marcoulides (Ed.), Modern methods for business research (p. 295336). Mahwah, New Jersey: Lawrence Erlbaum Associates.

Hair, J. F., Ringle, C. M., \& Sarstedt, M. (2011). PLS-SEM: indeed a silver bullet. Journal of Marketing Theory and Practice, 19(2), 139-151.

Hulland, J. (1999). Use of partial least squares (PLS) in strategic management research: a review of four recent studies. Strategic Management Journal, 20(2), 195-204.

Hußtegge, R. (2011). Selbstreguliertes Wollen als Bedingung für Studienerfolg an der Universität. Dissertation, Carl von Ossietzky Universität Oldenburg. http://oops. uni-oldenburg.de/1197/2/hussel112.pdf (12.11.2015).

KMK, Kultusministerkonferenz (2009). Hochschulzugang für beruflich qualifizierte Bewerber ohne schulische Hochschulzugangsberechtigung. Beschluss der Kultusministerkonferenz vom 06.03.2009.

Mayring, P. (2010). Qualitative Inhaltsanalyse. Grundlagen und Techniken. Weinheim: Beltz.

Marsh, H.W. \& O’Neill, R. (1984). Self-Description Questionnaire III (SDQ III): The construct validity of multidimensional self-concept ratings by late-adolescents. Journal of Educational Measurement, 21, 153-174.

Minks, K.-H., Netz, N. \& Völk, D. (HIS Hochschul-Informations-System GmbH, Hrsg.). (2011). Berufsbegleitende und duale Studienangebote in Deutschland. Status Quo und Perspektiven. http://www.his.de/pdf/pubfh/fh-201111.pdf. (12. 11.2015).

Nickel, S. \& Duong, S. (2012). Studieren ohne Abitur: Monitoring der Entwicklungen in Bund, Ländern und Hochschulen. CHE Centrum für Hochschulentwicklung: Gütersloh. 
Riggert S. C., Boyle M., Petrosko J. M., Ash D. \& Rude-Parkins C. (2006). Student employment and higher education: Empiricism and contradiction. Review of Educational Research, 76, 63-92

Ringle C. M., Sarstedt M. \& Straub D. W. (2012). A critical look at the use of PLSSEM in MIS Quarterly. MIS Quarterly, 36(1), iii-xiv.

Schiefele, U., Moschner, B. \& Husstegge, R. (2002). Skalenhandbuch SMILE-Projekt. Bielefeld: Universität Bielefeld, Abteilung für Psychologie.

Schiefele, U. , Streblow, L.; Ermgassen, U. \& Moschner, B. (2003). Lernmotivation und Lernstrategien als Bedingungen der Studienleistung. Ergebnisse einer Längsschnittstudie. Zeitschrift für pädagogische Psychologie, 17(3/4), 185-198.

Schuetze, H. G. \& Slowey, M. (2000). Higher Education and Lifelong Learners. International Perspectives on Change. London: Routledge.

Teichler, U. \& Wolter, A. (2004). Zugangswege und Studienangebote für nichttraditionell Studierende. Die Hochschule, 2004 (2), 64-80. http://www.hof.unihalle.de/journal/texte/04_2/Teichler_Zugangswege_und_Studienangebote.pdf (10.4.2016).

Thiel, F., Veit, S., Blüthmann, I., Lepa, S. \& Ficzko, M. (2008). Ergebnisse der Befragung der Studierenden in den Bachelorstudiengängen an der Freien Universität Berlin - Sommersemester 2008. http://www.fu-berlin.de/universitaet/entwicklung/qualitaetsmanagement/bachelorbefragung/bachelorbefragung2008.pdf?1304061426 (10.4.2016).

Wild, K.-P., Krapp, A., Schiefele, U., Lewalter, D. \& Schreyer, I. (1995). Dokumentation und Analyse der Fragebogenverfahren und Tests (Berichte aus dem DFGProjekt „Bedingungen und Auswirkungen berufsspezifischer Lernmotivation“, Nr. 2). München: Universität der Bundeswehr, Institut für Erziehungswissenschaft und Pädagogische Psychologie.

Wolter, A. (2000). Non-traditional students in German higher education - Situation, profiles, policies and perspectives. In H. G. Schuetze \& M. Slowey (Eds), Higher Education and Lifelong Learners. International Perspectives on Change (p. 4866). London: Routledge.

Wolter, A., Banscherus, U., Kamm, C. \& Otto, A. (2014). Durchlässigkeit zwischen beruflicher und akademischer Bildung als mehrstufiges Konzept: Bilanz und Perspektiven. Beiträge zur Hochschulforschung, 36(4), 8-39.

Wolter, A. \& Geffers, J. (2013). Zielgruppen lebenslangen Lernens an Hochschulen Ausgewählte empirische Befunde. https://de.offene-hochschulen.de/public_libraries/1 (12.11.2015).

Zawacki-Richter, O., von Prümmer, C. \& Stöter, J. (2015). Open Universities: Offener Zugang zur Hochschule in nationaler und internationaler Perspektive. Beiträge zur Hochschulforschung, 37(1), 8-25.

Zinn, B. (2012). Ein ingenieurwissenschaftliches Studium von beruflich qualifizierten Studierenden - Chancen und Risiken. Zeitschrift für Berufs- und Wirtschaftspädagogik, 108(2), 273-290. 